\title{
Stability Analysis of a Two-dimensional Tethered Helicopter
}

\author{
Matthew R. Parsons, Robert G. Langlois \\ Carleton University, Department of Mechanical and Aerospace Engineering \\ 1125 Colonel By Drive, Ottawa, Ontario, K1S 5B6, Canada \\ matthew.parsons@carleton.ca; robert.langlois@ carleton.ca
}

\begin{abstract}
Motivated by a haul down system used by ship borne helicopters, the objective of this paper was to analyze a planar helicopter attached to an earth-fixed tether to investigate the effect of the tether on the stability of the system. The model was developed without small angle approximations and without linearization to allow analysis at large displacements and tether angles. This work demonstrated consistency with the hypothesis that the addition of the tether could destabilize an otherwise stable system. The model presented in this paper established the foundation upon which a refined model, applicable to real helicopters, can be developed.
\end{abstract}

Keywords: helicopter, haul down system, tethered, shipborne

\section{Introduction}

Helicopters are intrinsically complex dynamic systems for which analysis tends to rely on approximations and linearizations. Often, a helicopter will have a cable attached to allow it to carry a load of cargo, to lower and raise personnel from a remote location, or as an aid in landing the helicopter onto the deck of a ship. Regardless of the function of the cable, the dynamic system is made even more complex as the tension vector of the cable will vary with oscillations of a free load, or with dynamics of the mechanism controlling tension in the cable and with helicopter motion and relative position when tethered to a fixed point. When the cable is a tether attached to a ship haul-down device, all of the considerations will affect the tension vector.

The haul down system is already known to stabilize the helicopter to some degree in that a lateral displacement away from the tether attachment point will create a lateral restoring force. However, since the tether must be attached to the belly of the helicopter, a lateral displacement can also create a moment that rolls the helicopter away from the attachment point, suggesting that there is a limit to the envelope where the haul down system provides stability. The potentially large displacement and correspondingly large cable angles invalidate the linearity and small angle assumptions that are often used in analysis of helicopter dynamic systems.

As an initial investigation into this stability envelope, a mathematical model of a helicopter tethered to a moving massive object is developed. The model is simplified by constraining its motion to a vertical plane. The helicopter can move laterally (left-right), vertically (up-down), and can roll (lean left-lean right). A real world helicopter was not used in this model, but parameters were identified based on common medium utility helicopters. Both analysis and simulation of the model are used to investigate the stability envelope.

\section{Modelling the System}

Modelling of this system was a balance between complexity and sufficient fidelity. The tension in the tether, the controllable lift vector of the helicopter, and the location of the ship over time were modelled with arbitrary functions to allow future development of those systems. The centre of lift, the centre of gravity, and the tether attachment point were chosen to be collinear along the vertical axis of the helicopter. The forces acting on the helicopter are shown in the diagram at Fig. 1, and all variables used are defined in Table 1. Four coordinate frames were necessary for this model,

- The earth-frame is fixed to an arbitrary position on a flat earth, with axes aligned to North, East and down. The course of the ship and the wind are represented in the earth-frame.

- The ship-frame is fixed to an average position of the deck of the ship. Ship motion other than the intended course of the ship, such as heave, roll, or sway, etc, is represented in the ship-frame.

- The helicopter-frame is centred on the helicopter centre of mass, and parallel to the ship frame. The tether and the relative wind (i.e., resultant wind from ship motion and surface wind as seen by the helicopter) effects are calculated in the helicopter-frame.

- The body-frame is centred on the helicopter centre of mass, with axes fixed to the helicopter structure. Moment arms and the lift vector are determined in the body-frame.

The state space equations will be generated within the ship-frame, so zero velocity will mean zero velocity relative to the course of the ship. 
Table 1: Description of variables.

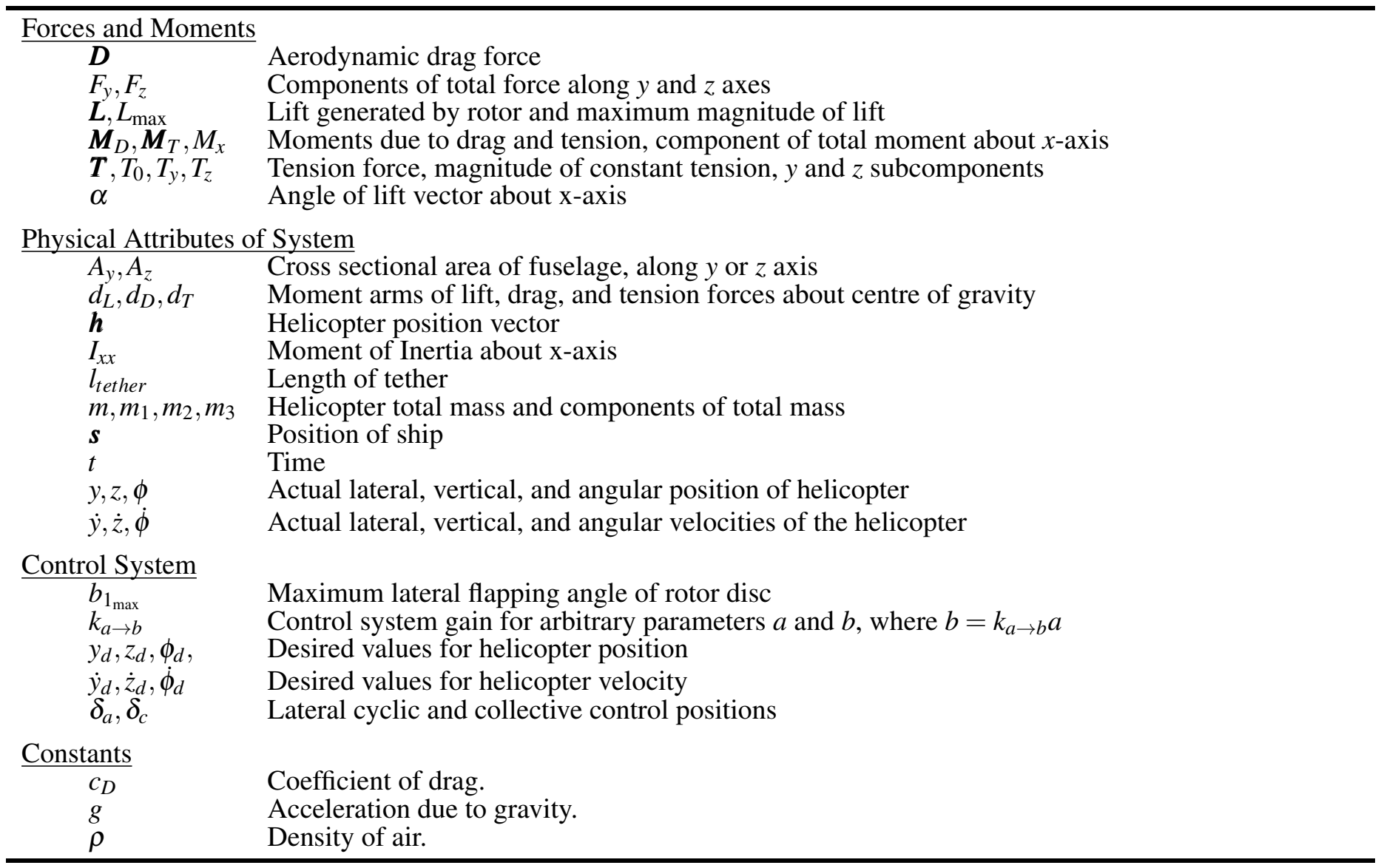

\subsection{Wind and Ship Motion}

This analysis uses only a non-moving ship model where the position of the ship is given as $\boldsymbol{s}(t)=0$. Because the planar helicopter model does not include any cross-axis rotor or control effects, forward motion of the ship will not affect the stability analysis. It is important to include lateral motion and heave of the ship when the pilot/controller workload or the safe operating envelope of the system is considered, but it will not provide any additional detail into this analysis on the effect of the tether.

A lateral velocity component of the wind will affect the stability analysis as it will have an effect on the control margins. Constant lateral wind is introduced to the system by adding the relative wind velocity to the helicopter velocity for any calculations that involve windspeed.

\subsection{Aerodynamic Effects}

Aerodynamic effects were modelled to include only the fuselage drag, using the drag

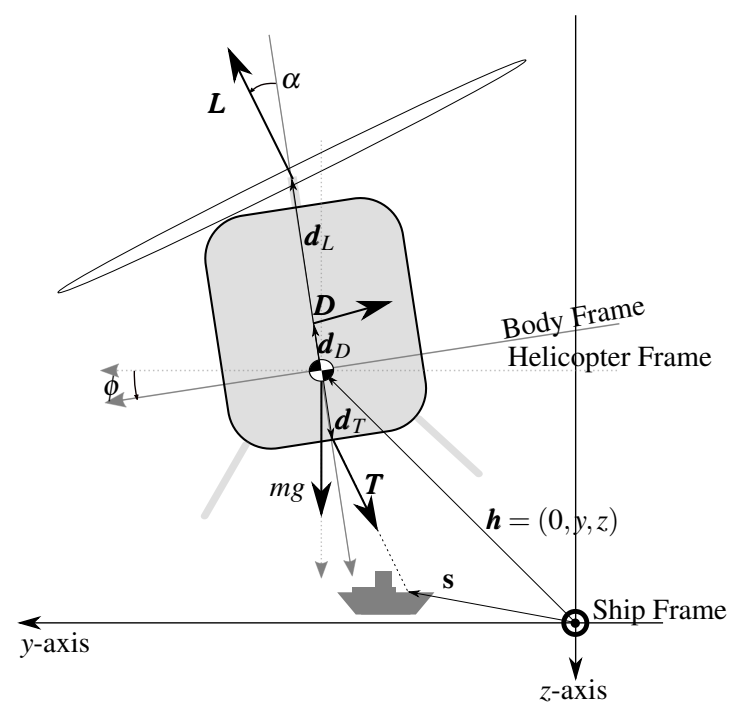

Fig. 1: System diagram. View is looking aft from the front of the helicopter, so $x$-axis is normal to the page. Earth-frame is not shown.

coefficient of a cube $\left(c_{D}=1.05\right)$ based on observations of typical medium utility helicopter cross-sections. Rotor effects were not included in the model because, although necessary for accurately modelling the stability of a specific helicopter, the analysis of the tether is a delta comparison for an arbitrary model which should be valid provided the untethered stability is acceptable. 


\subsection{Helicopter Model}

The helicopter geometric model is a rectangular solid. The mass model is built with three thin but massive plates lying parallel to the floor of the helicopter: one at the floor level to represent helicopter structure and fuel load, one at the roof to represent the rotor, transmissions, and upper helicopter structures, and the third is placed between the two and represents the cargo. The masses and the vertical location of the cargo are adjustable to allow variation of the vertical location of the centre of gravity.

\subsection{Haul Down System}

The haul down system is modelled as a massless cable connected at one end to a belly-mounted hook on the helicopter with a mechanical device at the ship end that applies tension in the cable and allows the helicopter to draw more cable as the helicopter moves away from the ship. For this analysis, the tension in the cable will be modelled with an ideal tension controller, producing constant tension, $T_{0}$, regardless of the helicopter position or velocity. Although this is an extremely demanding specification, and perhaps unrealistic, this choice was necessary to ensure any stability differences between the tethered and untethered conditions are a result of the tether vice the tether controller. Using the hook and ship locations to define the direction of the tension vector gives:

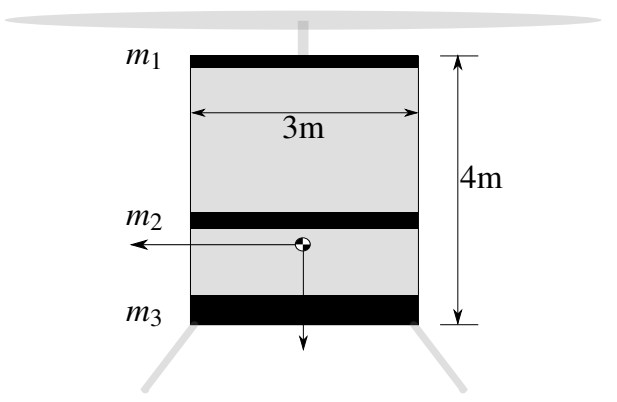

Fig. 2: Simplified mass model. Black regions represent locations of mass. Grey regions are massless structures of the modelled helicopter.

$$
\boldsymbol{T}=T_{0} \frac{\boldsymbol{s}-\left(\boldsymbol{h}+\boldsymbol{d}_{T}\right)}{\left|\boldsymbol{s}-\left(\boldsymbol{h}+\boldsymbol{d}_{T}\right)\right|}=T_{0} \frac{\boldsymbol{s}-\left(\boldsymbol{h}+\boldsymbol{d}_{T}\right)}{l_{\text {tether }}}=\left(0, T_{y}, T_{z}\right)
$$

With the ship location given as $\boldsymbol{s}(t)=0$, the tension becomes a function of angle of bank and helicopter position, or $\boldsymbol{T}=\left[0, T_{y}(y, \phi), T_{z}(z, \phi)\right]$. The moment that the tether produces will be about the $x$-axis and is found as the $x$ component of the cross product of the location of the hook (in the helicopter-frame) with the tension vector.

$$
\boldsymbol{M}_{T}=\boldsymbol{d}_{T} \times \boldsymbol{T}=\left[d_{T}\left(-T_{z} \sin \phi-T_{y} \cos \phi\right), 0,0\right]
$$

\subsection{Controller Variables}

As with a conventional helicopter, the single rotor in this planar model provides control of both lift and roll. Because the intent was not to create a reliable control system but was to investigate the effect of the tether, a simple linear feedback control was designed for each control variable $\left(\delta_{a}\right.$ and $\left.\delta_{c}\right)$. In order to correlate the results of the analysis with real-world effects, the controllers were inspired with the human pilot techniques detailed below, but did not include the control system equivalencies of a human pilot that appear in literature [1]. The responses to visual stimuli and the neuromuscular lag, to name just a few, are concepts that may have significant effects on the model, but are beyond the scope of this investigation.

\subsubsection{Vertical Position \& Velocity}

The human pilot would monitor both vertical location and vertical rate, and would adjust the collective to command a vertical rate that would attain the desired condition $\left(z_{d}\right.$ or $\left.\dot{z}_{d}\right)$. The position/rate combination allows the controller to acquire and maintain a height or to control rate during a take-off or a landing. The implemented height controller uses proportional feedback control on the height position error to command a correcting vertical velocity. That correcting vertical velocity is summed with a vertical velocity error and scaled to produce $\delta_{c}$, the collective position.

$$
\delta_{c}=k_{\dot{z} \rightarrow \delta_{c}}\left[k_{z \rightarrow \dot{z}}\left(z_{d}-z\right)+\left(\dot{z}_{d}-\dot{z}\right)\right]
$$

\subsubsection{Lateral Position \& Velocity}

Similar to the vertical case, the human controller would correct lateral position errors by commanding a lateral velocity, and would correct lateral velocity errors also by commanding a velocity. However, in a typical helicopter cockpit, a lateral cyclic input does not directly command a lateral velocity. Lateral cyclic adjusts the direction of the lift vector of the helicopter rotor disc, creating a lateral force component that will accelerate the helicopter somewhat, but more significantly will create an angular acceleration. A lateral force with no angular acceleration is achieved by setting an angle of bank for the entire helicopter, and thus the rotor lift vector, with the lateral cyclic near a neutral or trim position. For constant lateral velocity, an angle of bank must be found where the lateral forces balance.

That real world behaviour is modelled in this controller by having lateral position error command a velocity. The commanded velocity is summed with a velocity error, which becomes the source for the correcting angle of bank (i.e., 
correcting the position error). The correcting bank angle is summed with the bank angle error and scaled to produce a correcting angular rate, which is then summed with an angular rate error and scaled to determine the lateral cyclic $\left(\delta_{a}\right)$ position.

$$
\delta_{a}=k_{\dot{\phi} \rightarrow \delta_{a}}\left[k_{\phi \rightarrow \dot{\phi}}\left[k_{\dot{y} \rightarrow \phi}\left[k_{y \rightarrow \dot{y}}\left(y_{d}-y\right)+\left(\dot{y}_{d}-\dot{y}\right)\right]+\left(\phi_{d}-\phi\right)\right]+\left(\dot{\phi}_{d}-\dot{\phi}\right)\right]
$$

Finally, the inceptor values need to be converted into forces and moments. Observations of medium utility helicopters provided the maximum value of lift, $L_{\max }$, and the maximum lateral flapping angle of the disc, $b_{1 \max }{ }^{1}$. The conversion from control input to a physical value is given as: $\mathrm{rCl} \mathrm{L}(\mathrm{z})=,\delta_{c} L_{\max }$

$\alpha(y, \phi, \dot{y}, \dot{\phi})=\delta_{a} b_{1_{\max }}$

\subsection{System Dynamics}

The system dynamics are determined by summing the forces and moments exerted on the helicopter and applying the Newton-Euler formulation: $\operatorname{rlCCCL} \sum F_{y}=m \ddot{y}=L \sin (\alpha+\phi)-T_{y}-\frac{1}{2} \rho c_{D} A_{y} \dot{y}|\dot{y}|$

$\left\{[10] \sum F_{z}=m \ddot{z}=-L \cos (\alpha+\phi)+T_{z}-\frac{1}{2} \rho c_{D} A_{z} \dot{z}|\dot{z}|+m g\right.$

$\sum M_{x}=I_{x x} \ddot{\phi}=d_{L} L \sin \alpha+M_{T}+M_{D}$ where $\boldsymbol{M}_{D}=\boldsymbol{d}_{D} \times \boldsymbol{D}$ is the moment that is a result of the drag forces acting at the centre of the planform area, which is not necessarily colocated with the centre of gravity.

\section{Stability Analysis}

The analysis of the stability of this system ultimately has two desirable outcomes: to predict whether the helicopter will remain stable once the tether is attached and to establish upper bounds on parameters to define where stability can be assured. An intermediate step towards the latter outcome is to define limits on the parameters beyond which the system is known to be unstable. An upper bound of that box is investigated using a mathematical analysis of the dynamic system in Section 3.1, and the effect of adding a tether to a stable system is investigated through simulation in Section 3.2.

\subsection{Control Limits}

Both the magnitude and the direction of the lift vector are bounded by manufactured limitations of a helicopter as $\left\{L \mid 0 \leq L \leq L_{\max }\right\}$ and $\left\{\alpha \mid-b_{1_{\max }} \leq \alpha \leq b_{1_{\max }}\right\}$. These limits create an upper bound on the lateral force the rotor provides and with that, a limit on the moment about the $x$-axis that can be commanded, for a given helicopter position and roll angle. In static equilibrium, the net forces and moment become zero, the drag becomes zero and with values for the tension components included, Equation 2.6 becomes:

$$
\begin{gathered}
\text { rlCL } 0=\mathrm{L} \sin (\alpha+\phi)-\frac{T_{0}}{l_{\text {lether }}(y, z, \phi)}\left(-y+d_{T} \sin \phi\right)-0 \\
\left\{[10] 0=-L \cos (\alpha+\phi)+\frac{T_{0}}{l_{\text {tether }}(y, z, \phi)}\left(-z-d_{T} \cos \phi\right)-0+m g\right. \\
0=d_{L} L \sin \alpha+\frac{d_{T} T_{0}}{l_{\text {tether }}(y, z, \phi)}(z \sin \phi+y \cos \phi)+0
\end{gathered}
$$

By choosing a height and selecting $\alpha= \pm b_{1_{\max }}$, a relationship between $L, y$, and $\phi$ would describe an upper bound to the a region of static stability. A numerical analysis of this was completed but the plots were uninteresting in that they did not identify any bounds for the region of instability, until parameters such as maximum flapping angle, magnitude of the tension, or the vertical centre of gravity were altered to the extent that the model was no longer realistic. This was still a useful result in that it confirmed that a bound does exist, but one that is only of potential value once an accurate model is determined.

\subsection{Simulation}

The dynamic system was implemented in MATLAB [2] and Simulink [3]. The parameters used to model the helicopter are listed in Table 2. To ensure that unrealistic flight conditions were not demanded, saturation effects were included with the calculation of each condition and with each inceptor value. The simulation was modelled in the ship-frame so the "on-top" position of the helicopter was defined as $(y, z, \phi)=(0,-10 \mathrm{~m}, 0)$. Stability was demonstrated using four manoeuvres that used different initial conditions and required the helicopter be flown to the on-top position and one manoeuvre that had the helicopter move away from the ship to a laterally displaced position. The manoeuvres are listed at Table 3.

Seven different wind conditions were used $(0,3,7,15,20$, and $25 \mathrm{~m} / \mathrm{s})$, all from the right side of the helicopter, so appearing to the system as a positive velocity. Each pairing of manoeuvre and wind condition was flown both with and without a tether, creating seventy unique test conditions. Note that a $25 \mathrm{~m} / \mathrm{s}$ crosswind is well outside the envelope of a conventional helicopter. This was a necessary inclusion to ensure the effect of the lateral wind was seen in the simulation. The requirement for such high wind speeds arose from the estimation of helicopter geometry rather than using an existing machine and thus, realistic winds.

A crash condition was specified for the simulation that included a minimum and a maximum allowable value for each component of the state vector. The values typically exceeded the conditions in which a real helicopter would operate, allowing

\footnotetext{
${ }^{1}$ The variable $b_{1_{\max }}$ comes from the coefficient of the first cosine term of the Fourier expansion of the pitch angle of a three-dimensional rotor blade.
} 
Table 2: Parameters that define the helicopter model.

\begin{tabular}{|c|c|c|c|c|c|}
\hline \multirow{2}{*}{$\frac{\text { Helicopter Geometry }}{\text { Length ( } x \text {-axis) }}$} & \multicolumn{3}{|c|}{ Saturation Limits } & \multicolumn{2}{|c|}{ Control Gains } \\
\hline & $10 \mathrm{~m}$ & Lateral Position Correction & $\pm 10 \mathrm{~m}$ & $k_{y \rightarrow \dot{y}}$ & $0.3 / \mathrm{s}$ \\
\hline Width $(y$-axis) & $3 \mathrm{~m}$ & Lateral Velocity Correction & $\pm 10 \mathrm{~m} / \mathrm{s}$ & $k_{\dot{\dot{y}} \rightarrow \phi} \rightarrow y$ & $3 \% / \mathrm{m} / \mathrm{s}$ \\
\hline Height (z-axis) & $4 \mathrm{~m}$ & Roll Angle Correction & $\pm 45^{\circ}$ & $k_{\phi \rightarrow \phi}$ & $1.0 / \mathrm{s}$ \\
\hline Helicopter Mass & $5000 \mathrm{~kg}$ & Roll Rate Correction & $\pm 45 \% / \mathrm{s}$ & $k_{\dot{\phi} \rightarrow \delta_{\theta}}^{\varphi \rightarrow \psi}$ & $2.22 \% 1^{\circ}$ \\
\hline & & Collective & $0-100 \%$ & $k_{z \rightarrow \dot{z}}$ & $1.0 / \mathrm{s}$ \\
\hline Moment Arms & & Lateral Cyclic & $\pm 100 \%$ & $k_{\dot{z} \rightarrow \delta_{c}}$ & $20 \% / \mathrm{m} / \mathrm{s}$ \\
\hline $\begin{array}{l}d_{D} \\
d_{T}\end{array}$ & $\begin{array}{l}0.6 \mathrm{~m} \\
1.4 \mathrm{~m}\end{array}$ & Maximum Control Values & & & \\
\hline$d_{L}$ & $2.6 \mathrm{~m}$ & $\begin{array}{l}L_{\max } \\
b_{1_{\max }}\end{array}$ & $\begin{array}{l}60 \mathrm{kN} \\
12^{\circ}\end{array}$ & & \\
\hline
\end{tabular}

Table 3: Manoeuvres flown by the helicopter.

\begin{tabular}{cl}
\hline Manoeuvre & Description \\
\hline$\# 1$ & $\begin{array}{l}\text { Start } 1 \mathrm{~m} \text { below and } 1 \mathrm{~m} \text { right of on-top position, return to on-top position. This is a gentle } \\
\text { correction that demonstrated the effect of small tether angles. }\end{array}$ \\
Start $10 \mathrm{~m}$ right of on-top position, return to on-top position. This is a role relevant manoeuvre \\
that should demonstrate stability in any realistic wind conditions. \\
Start $50 \mathrm{~m}$ right of on-top position, return to on-top position. This is an overly-large offset and is \\
intended to demonstrate the effect of large tether angles.
\end{tabular}

observation of unusual flight behaviours, but certain bounds were necessary to avoid non-representative flight such as flight below the surface of the earth or inverted, tethered flight.

\subsubsection{Untethered Performance}

The untethered helicopter was able to achieve the desired end-state in all manoeuvres and wind conditions, except for the $25 \mathrm{~m} / \mathrm{s}$ wind when flying manoeuvre \#5. Fig. 3 shows that the correction of a $50 \mathrm{~m}$ offset to the on-top position took 20 seconds in a $0 \mathrm{~m} / \mathrm{s}$ wind with two lateral overshoots, suggesting a realistic but somewhat aggressive pilot. The helicopter drops initially, and then does not recover fully to the desired height. The drop is due to the additional power required to move the helicopter, and the steady-state error is the nature of the proportional controller. Because the desired condition is applied instantly at time $t=0$, the error in the system behaves as a step input, for which a proportional controller will generate a steady-state error [4]. Although the small error could have been corrected with an improved controller, the system was left with this known deficiency because the intent was to keep the overall model as simple as possible yet representative of a human-piloted aircraft. Additionally, the error was small enough to consider the response to be representative of true pilot behaviour.

\subsubsection{Tethered Performance - Height Control}

With a tether added, the height channel response was similar to the untethered height channel response for most of the test conditions, except that the steady state error was approximately $0.5 \mathrm{~m}$ lower, as shown in Fig. 4 . The constant tension in the cable creates a greater downwards force as an input to the controller, which then generates a greater steadystate error. This response would not be anticipated with a real pilot as there would be awareness of the tether and the pilot would compensate. However, the error remains sufficiently small that the tethered height performance is representative of a human-piloted manoeuvre. 


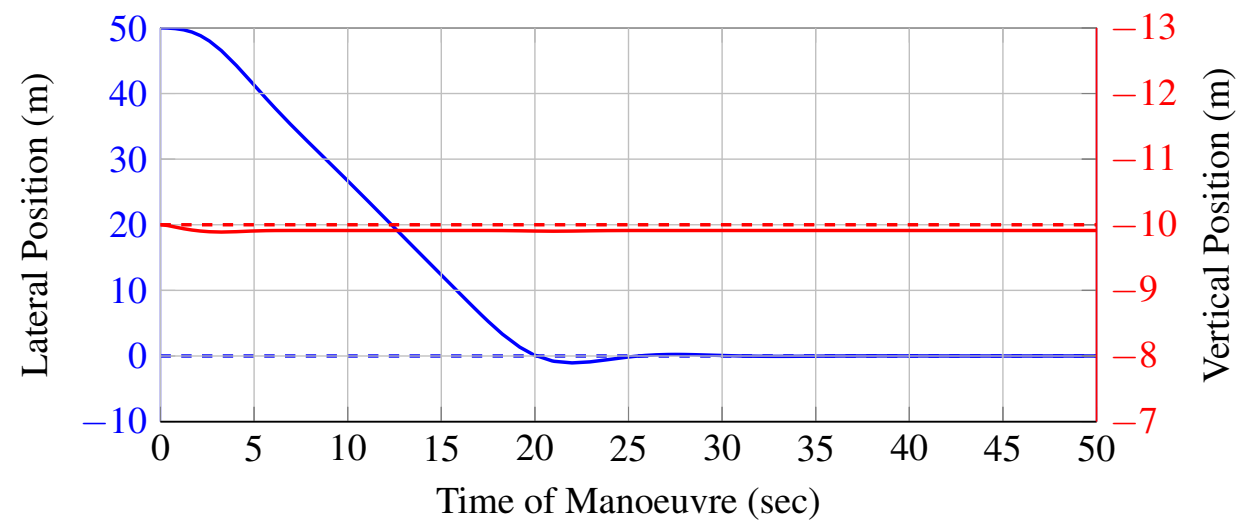

Fig. 3: Reposition from $50 \mathrm{~m}$ offset with no tether and zero wind showing lateral and vertical position over time.

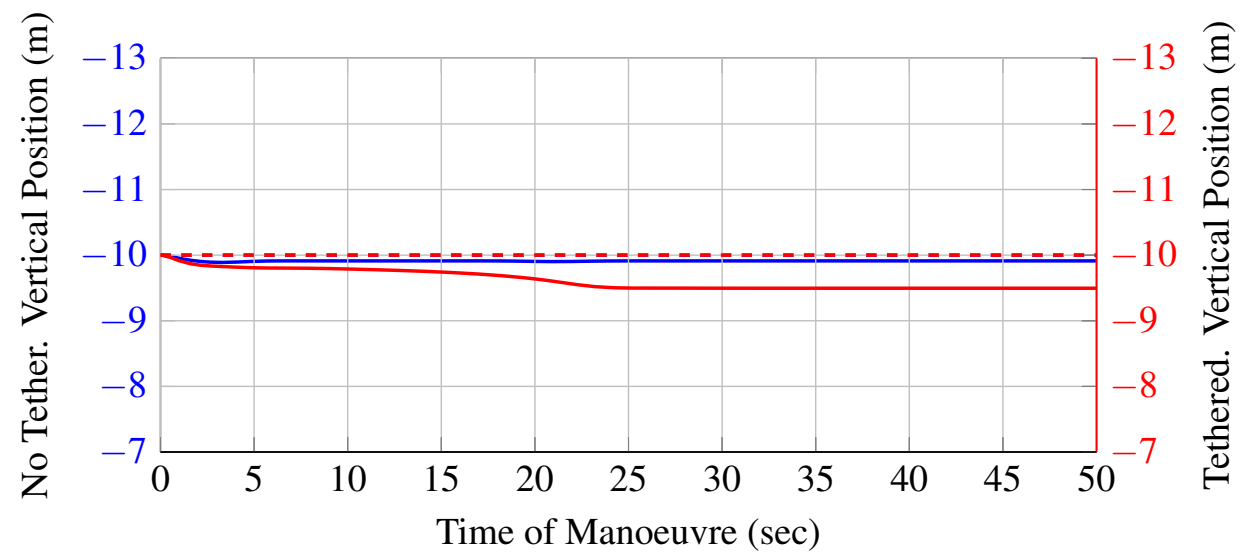

Fig. 4: Reposition from $50 \mathrm{~m}$ offset with zero wind comparing vertical position for untethered and tethered helicopter.

\subsubsection{Maximum Flapping Angle}

Wherever the control system was expected to be challenged by the manoeuvre or by the wind condition, the control position traces were studied. It was unusual, even in the most demanding test conditions, for the lateral cyclic to exceed $\pm 20 \%$ of its maximum value. The maximum values for lateral cyclic would depend on the maximum flapping angle, $b_{1_{\max }}$, even though it is not embedded within the controller equations. This is due to the output of the controller being scaled by $b_{1_{\max }}$, which affects the response of the dynamic system and causes the controller to vary the control position accordingly. Thus, the low values of lateral cyclic position were assumed to be a result of an overly large maximum flapping angle. This is similar to the result of the control limit analysis in Section 3.1 and model improvement must remain an area for further development.

To ensure an artificially-high amount of control power was not provided to the lateral cyclic, different values of $b_{1_{\max }}$ were evaluated under all seventy test conditions. Fig. 5 shows the lateral position of the helicopter flying manoeuvre \#3, with a tether, in $25 \mathrm{~m} / \mathrm{s}$ winds with $b_{1_{\max }}=12^{\circ}, 10^{\circ}, 8^{\circ}$, and $6^{\circ}$. Fig. 6 shows lateral cyclic position for the same conditions. The lateral position demonstrates a 'cliff edge' sensitivity to the maximum flapping angle in that the extrapolation of the three larger maximum flapping angles does not predict the smallest one. The lateral cyclic position shows lightly damped oscillatory motion between 0 and 25 seconds, when the helicopter was out of position and a lateral velocity was to be commanded. After 25 seconds, the helicopter should have decelerated until the on-top position was attained. Instead, the controller gradually increased lateral cyclic up to the maximum despite lateral velocity continuing to increase. With a $6^{\circ}$ maximum flapping angle, the tethered helicopter was unable to control lateral velocity and thus was unable to maintain position. For comparison, the untethered helicopter achieved the desired conditions under all maximum flapping angles, but did show undamped oscillatory motion once stabilized at the on-top position with a $6^{\circ}$ maximum flapping angle. 


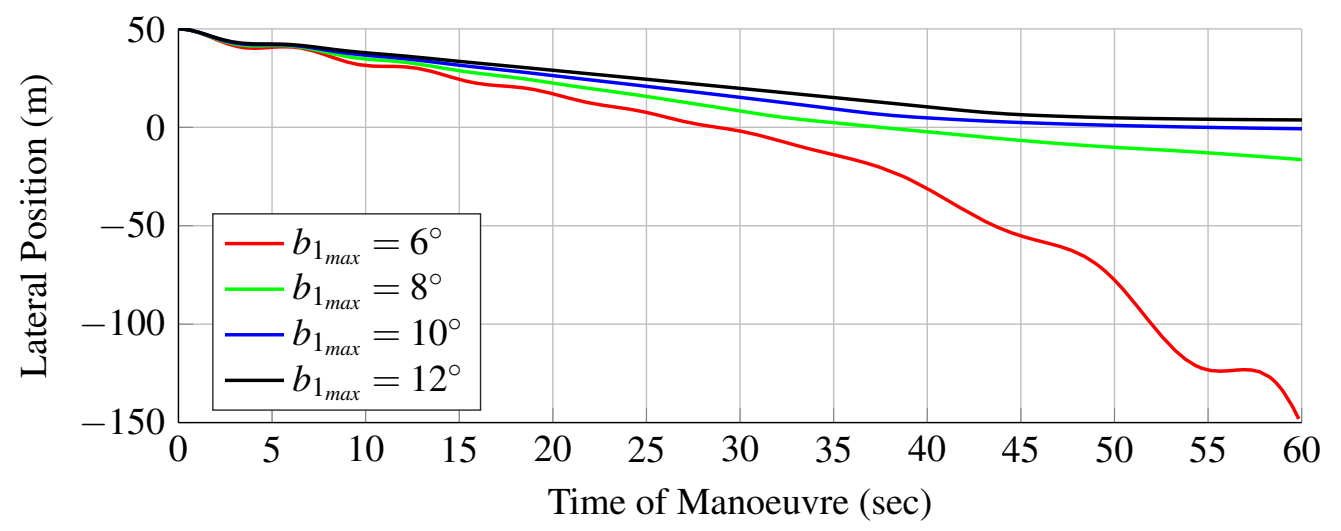

Fig. 5: Lateral position during $50 \mathrm{~m}$ reposition, tethered, in $25 \mathrm{~m} / \mathrm{s}$ wind with various maximum flapping angles.

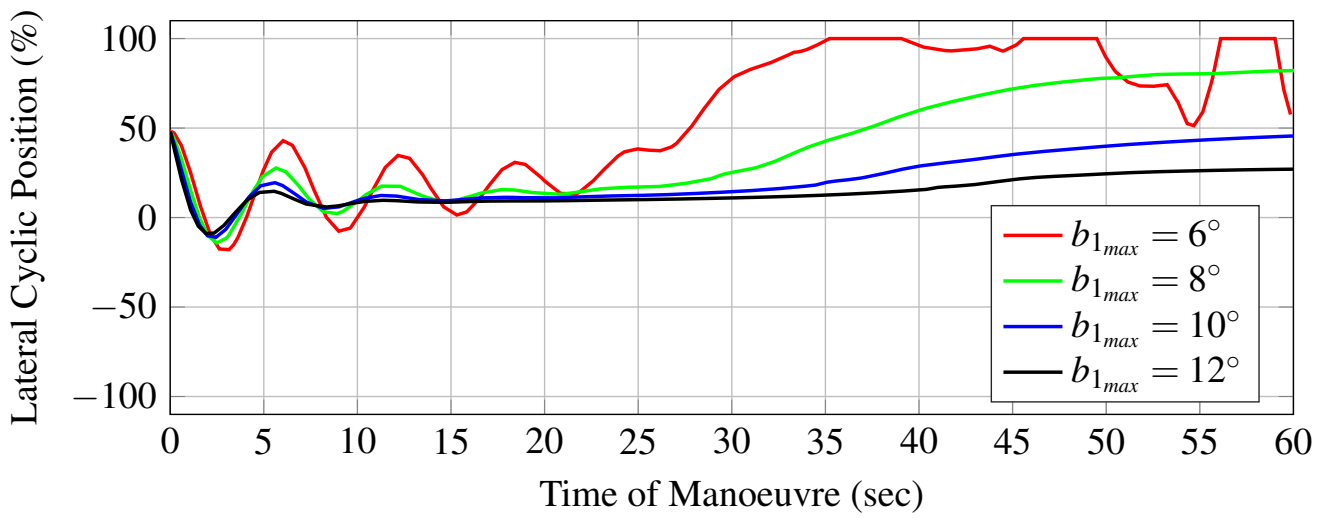

Fig. 6: Control positions during a $50 \mathrm{~m}$ reposition, tethered, in $25 \mathrm{~m} / \mathrm{s}$ wind with various maximum flapping angles.

\subsubsection{Excess Power Demand}

Three of the seventy test conditions using the original $b_{1_{\max }}=12^{\circ}$ resulted in crashes. Both the tethered and the untethered helicopters crashed during manoeuvre \#5 in $25 \mathrm{~m} / \mathrm{s}$ winds and only the tethered helicopter crashed on the same manoeuvre with winds reduced to $20 \mathrm{~m} / \mathrm{s}$. Because this manoeuvre in these winds was known to be very challenging, these crashes helped to validate the model in that a real helicopter likely would have crashed if attempting this condition.

With the winds reduced to $20 \mathrm{~m} / \mathrm{s}$, the untethered helicopter flew the manoeuvre to the desired conditions in approximately 20 seconds, but the tethered helicopter crashed after 5 seconds. Fig. 7 shows the vertical and lateral position of the helicopter, and Fig. 8 shows the cockpit control inputs. The helicopter height reduced to $2 \mathrm{~m}$ above the surface and triggered the crash condition. The roll angle needed to be large to generate lateral forces strong enough to decelerate the helicopter, to counter the crosswind and to translate to the desired position. The tether was creating a moment that rolled the helicopter away from the ship, so was helping the rotational control, but was also adding down-force and thus an increased lift requirement. With the roll angle approaching $45^{\circ}$, the vertical component of the lift vector decreased with the cosine of that angle so even at maximum collective position there was insufficient power to maintain flight. This behaviour with the tether is a very important consideration, but is not the tether-induced instability that the analysis was investigating.

\section{Conclusion}

This analysis used a non-linear model of a dynamic system representing a helicopter but did not include any of the effects of the helicopter rotor. Parameters obtained through observation and experience in the real operating environment developed the model into one that demonstrated responses consistent with a real model piloted by a human. Improvements to the model will be required before conclusive results about a particular model can be made.

The limits of static stability could not be found without significant changes to the model, but two different dynamic effects were observed where the addition of the tether created an instability. The first was related to the increased power requirement due to the tether increasing the total downforce. This was not the intended observation of this analysis, but as it is a known consideration, it added validity to the model. The second instability observed was related to the reduction of the maximum flapping angle, within a realistic domain. The tethered helicopter required more lateral force than was available 


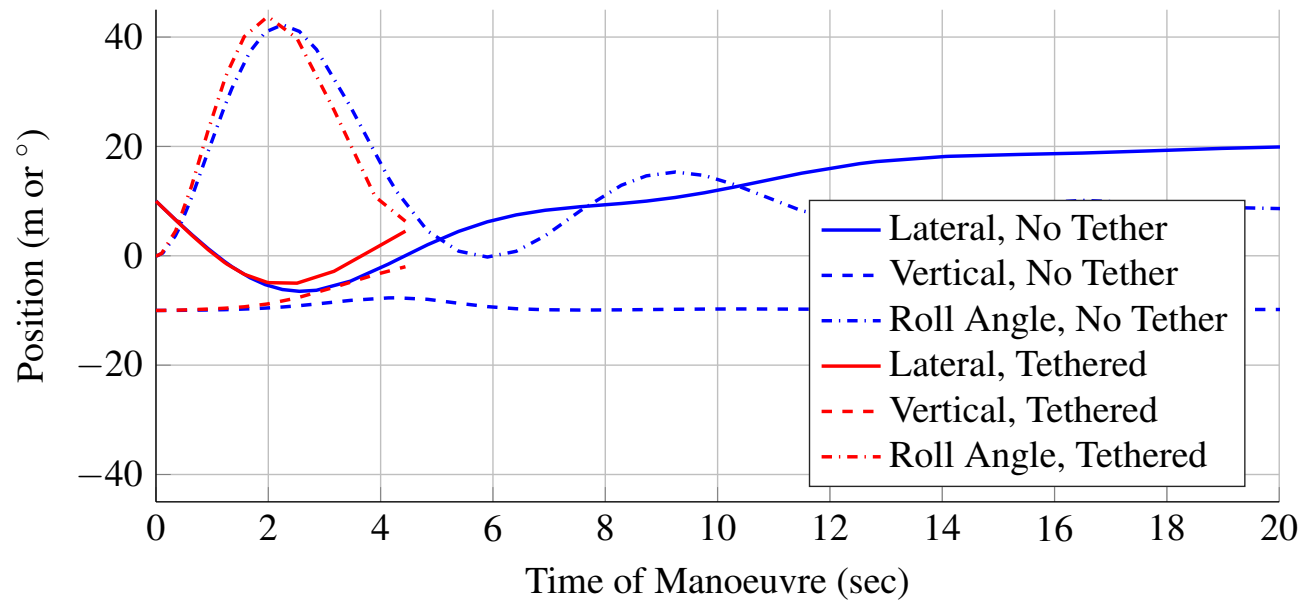

Fig. 7: Wave-off with $20 \mathrm{~m} / \mathrm{s}$ wind showing lateral \& vertical positions for untethered and tethered helicopter.

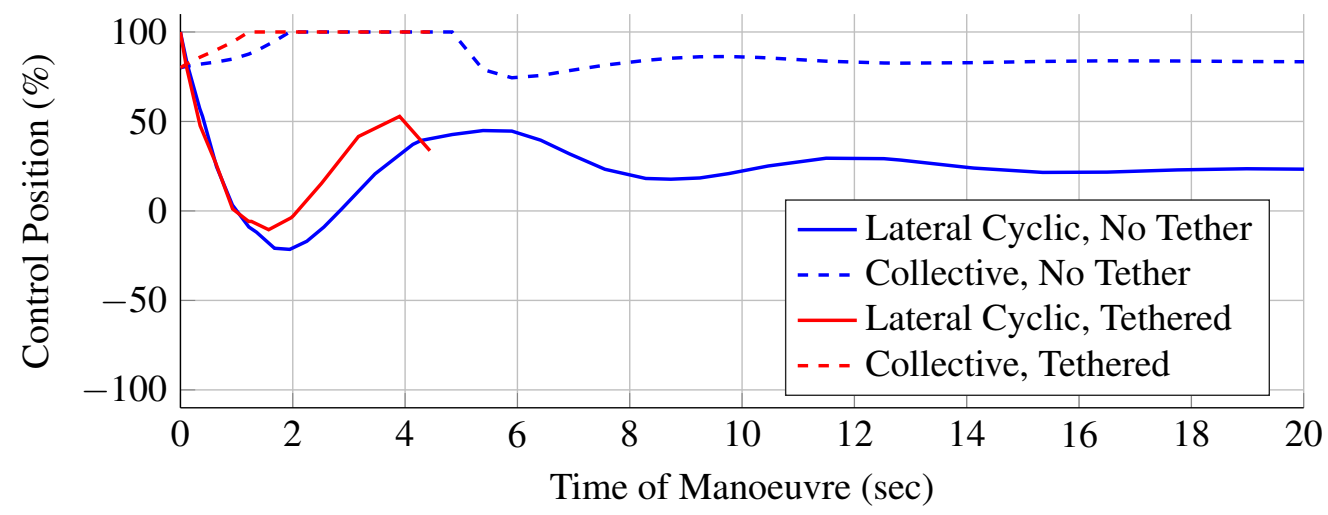

Fig. 8: Wave-Off with $20 \mathrm{~m} / \mathrm{s}$ wind showing lateral cyclic \& collective positions for untethered and tethered helicopter.

and was unable to arrest the lateral velocity it generated.

As predicted, addition of the tether to a stable helicopter flying a prescribed manoeuvre did result in unstable behaviour in certain regimes. The bounds of the stable regions for both the static and the dynamic stability were dependant on the parameters that define the model; so although the result has been verified, further work with a refined model is recommended.

\section{References}

[1] D. T. Mcruer and E. S. Krendel, "Mathematical models of human pilot behavior," NATO Science and Technology Organization, AGARD-AG-188, 1974.

[2] MATLAB Release 2015b, Natick, Massachusetts, United States: The MathWorks, Inc., 2015.

[3] Simulink Release 2015b Natick, Massachusetts, United States: The MathWorks, Inc., 2015.

[4] K. Ogata, Modern control engineering, 5th Ed. Boston: Prentice-Hall, 2010. 